\title{
Motor outcomes of repetitive transcranial magnetic stimulation are dependent on the specific interneuron circuit targeted
}

\author{
Ricci Hannah, Martin Sommer, Sean Cavanagh, Steven Jerjian, and John C Rothwell
}

\begin{abstract}
Different aspects of motor behaviour may engage distinct interneuron circuits in the human motor cortex. If so, the behavioural effects of repetitive transcranial magnetic stimulation (rTMS) protocols may critically depend on the specific circuit stimulated. We used TMS of the hand area to activate two distinct synaptic inputs to corticospinal neurons by altering the direction of current induced in the brain: posterior-anterior (PA inputs) and anterior-posterior (AP inputs). We found AP inputs to be preferentially suppressed during motor preparation in a reaction time task. We also show that preconditioning PA, but not AP, inputs with via rTMS facilitates performance of a ballistic motor task. These results suggest that behavioural effects of rTMS may be most evident when relevant interneuron circuits are targeted.
\end{abstract}

\section{INTRODUCTION}

$\mathrm{T}$ HERE is good evidence in healthy populations that repetitive TMS (rTMS) can have lasting effects on brain excitability and that these can occur alongside changes in simple behaviours. A given rTMS protocol is often assumed to interact with all motor behaviours equally, yet this may not be the case. Instead, the behavioural outcome might be influenced by the particular neuronal populations stimulated. Here we sought evidence to support this hypothesis.

It is well known that the response to a single TMS pulse over the primary motor cortex (M1) is sensitive to direction of the induced current in the brain [3]. Posterior-anterior pulses (PA) recruits motor evoked potentials (MEPs) with a lower intensity and shorter latency than anterior-posterior (AP) pulses. These differences are presumed to reflect recruitment of distinct excitatory inputs to corticospinal neurons. Until now, the behavioural relevance of these inputs had been largely unexplored.

In a recent study, we found that a paired-associative stimulation (PAS) protocol that putatively preconditioned PA-sensitive inputs facilitated learning of a ballistic motor task, whereas performance was unaffected by targeting APsensitive inputs [5]. A protocol putatively preconditioning AP-sensitive inputs, however, impaired learning of a visuomotor adaptation task, whilst preconditioning PA inputs had no effect. The implication is that these two inputs play specific roles in different forms of motor learning and that only by targeting the appropriate inputs do we see any

R. Hannah and J.C. Rothwell were supported by a Medical Research Council grant (MR/K01384X/1).

R. Hannah (r.hannah@ucl.ac.uk), S. Jerjian, S. Cavanagh, and J.C. Rothwell are with the Sobell Department of Motor Neuroscience and Movement Disorders, UCL Institute of Neurology, UK. M. Sommer is with the Department of Clinical Neurophysiology, University of Göttingen, Germany. behavioural effect.

The aim of the present experiments was to provide more direct evidence that PA and AP inputs play distinct roles in motor behavior by showing: (i) that their excitability is differentially modulated during a motor task; and (ii) that directly preconditioning each set of inputs produces specific effects on simple motor performance. To do this, we used a novel controllable pulse parameter TMS (cTMS) device [7]. We previously found that using monophasic AP pulses of short duration $\left(30 \mu \mathrm{s} ; \mathrm{AP}_{\mathrm{S}}\right)$ and long duration PA pulses $\left(120 \mu \mathrm{s} ; \mathrm{PA}_{\mathrm{L}}\right)$ more reliably activate different inputs [2]. This allows stimulation to be focused more clearly on different sets of inputs during single pulse and rTMS.

\section{MATERIAL AND METHODS}

Forty-five healthy participants participated in three different experiments (15 in each).

\section{A. Transcranial magnetic stimulation (TMS)}

Single pulse TMS and an intermittent theta burst stimulation (iTBS; [6]) protocol, a rapid method of conditioning the motor cortex, were delivered over the cortical representation of the first dorsal interosseous (FDI) muscle of the dominant hand via a cTMS device (Rogue Resolutions Ltd., UK), or a Magstim 200² (Magstim Co. Ltd., UK), connected to a figure-of-eight coil. MEPs were recorded via surface EMG of the FDI muscle.

\section{B. Experiment 1: Excitability of $P A$ and AP inputs during $a$ choice-reaction time task}

Previous research found MEPs were suppressed during the warning period of a choice reaction time task (CRTT) [4], perhaps to prevent premature responding. We tested whether this suppression was "global" or specific to PA or AP inputs by evaluating changes in AP- and PA-evoked MEP amplitude throughout the task. Participants received a visual warning (WS) $500 \mathrm{~ms}$ prior to a visual left/right imperative signal (IS) and then responded by flexing the index finger of the indicated hand. Twenty TMS pulses were delivered at the time of the WS, 250ms after WS, IS and 35 and $70 \%$ into each individual's reaction time (RT).

\section{Experiment 2: Direction-specific effects of rTMS on $M E P S$}

Monophasic iTBS was applied over the motor cortex with $\mathrm{PA}_{\mathrm{L}}$ and $\mathrm{AP}_{\mathrm{S}}$ pulses to assess its effects on standard MEPs (PA-oriented pulses via a Magstim $200^{2}$ device). MEPs were measured for 30 minutes post-iTBS. 
D. Experiment 3: Direction-specific effects of rTMS on motor performance

Monophasic iTBS was applied over the motor cortex $\left(\mathrm{AP}_{\mathrm{S}}\right.$ and $\mathrm{PA}_{\mathrm{L}}$ ) and a control site (vertex, $\mathrm{V}_{\mathrm{S}}$ ) in order to precondition the excitability of the AP and PA inputs. We evaluated its effect on CRTT performance and a ballistic finger tapping task, which required participants to rapidly flex/extend the first digit as many times as possible in $15 \mathrm{~s}$. Motor performance was assessed for 30 mins after iTBS.

\section{RESULTS}

\section{A. Experiment 1: Excitability of PA- and AP-inputs during a choice-reaction time task}

During the CRTT, $\mathrm{AP}_{\mathrm{S}}$-evoked MEPs were suppressed at the time of the IS and 35\%RT in the responding hand, whilst $\mathrm{PA}_{\mathrm{L}}$-evoked MEPs were unaffected (rmANOVA: pulse type $\times$ time interaction, $P=0.002$; Fig. 1 A).

B. Experiment 2: Direction-specific effects of rTMS on $M E P s$

The effect of iTBS on MEP amplitude was affected by pulse type (rmANOVA: pulse type $\times$ time interaction, $P<$ 0.002; Fig. 1B), with $\mathrm{AP}_{\mathrm{S}}$ pulses inhibiting and $\mathrm{PA}_{\mathrm{L}}$ pulses having minimal effect on MEPs.

\section{Experiment 3: Direction-specific effects of rTMS on motor performance}

The effect of iTBS on ballistic tapping performance was also dependent on pulse type (rmANOVA: pulse type $\times$ time interaction, $P=0.024$; Fig. $1 \mathrm{C}$ ). $\mathrm{PA}_{\mathrm{L}}$ iTBS facilitated finger tapping rate, whilst there was no significant effect of $\mathrm{AP}_{\mathrm{S}}$ iTBS or $V_{S}$ iTBS. Performance in the CRTT was unaffected by all three iTBS conditions (not shown).

\section{DISCUSSION}

Experiment 1 demonstrated that the excitability of APsensitive inputs is preferentially modulated during performance of a simple motor task, suggesting that AP and PA inputs play distinct roles in motor preparation and execution. To test this idea more directly, we then showed: (i) that targeting PA and AP inputs with monophasic iTBS protocol produces distinct effects on corticospinal excitability, suggesting a differential role of distinct interneuron circuits in the physiological response to rTMS; and (ii) that selectively preconditioning PA- and AP-inputs with iTBS produces distinct effects on motor performance, with $\mathrm{PA}_{\mathrm{L}}$ iTBS improving and $\mathrm{AP}_{\mathrm{S}}$ iTBS tending to impair ballistic tapping performance. The latter findings could explain why previous research found no effect of standard iTBS utilizing biphasic (PA-AP) pulses on a similar ballistic motor task [1].

\section{CONCLUSION}

Behavioural outcomes of rTMS appear sensitive to the pulse direction and thus the interneuron circuit stimulated.
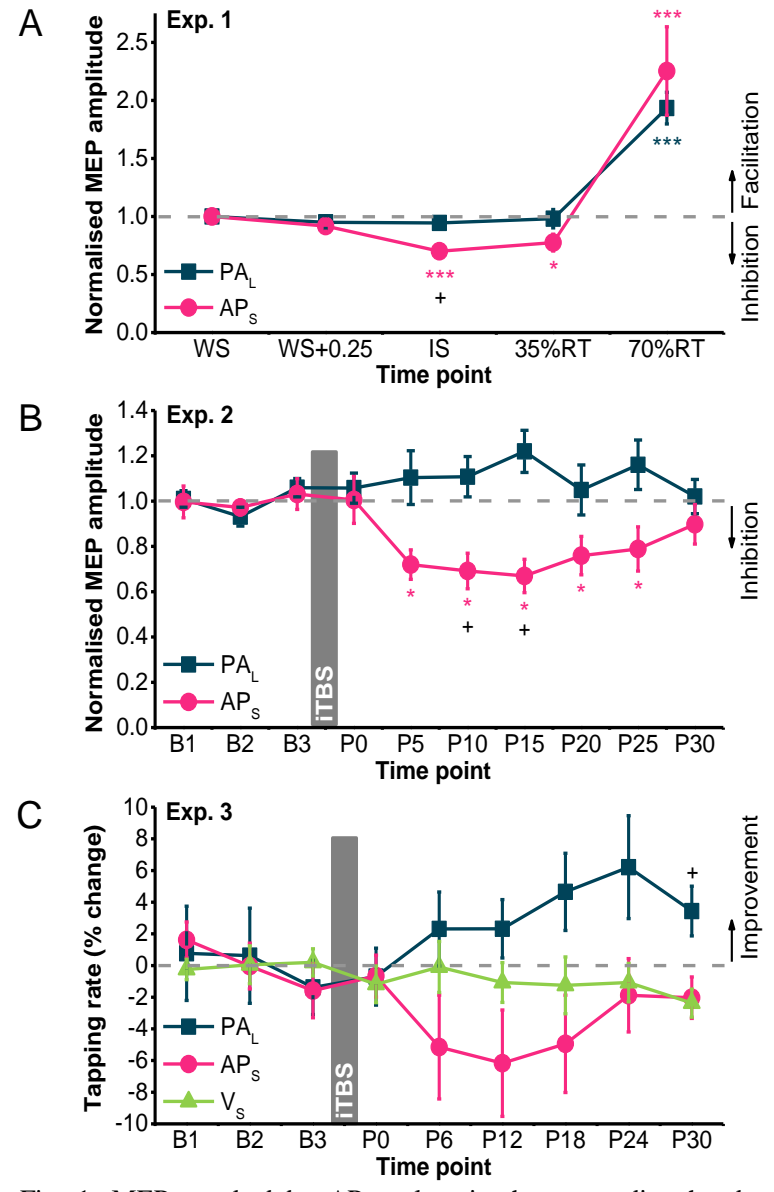

Fig. 1. MEPs evoked by $\mathrm{AP}_{\mathrm{S}}$ pulses in the responding hand were suppressed at the IS and 35\%RT during CRTT (A). iTBS delivered to motor cortex with $\mathrm{AP}_{\mathrm{S}}$ pulses suppressed MEPs, whereas $\mathrm{PA}_{\mathrm{L}}$-evoked MEPs tended to facilitate them (B). iTBS delivered to motor cortex with $\mathrm{PA}_{\mathrm{L}}$ pulses improved, and $\mathrm{AP}_{\mathrm{S}}$ pulses tended to impair, rapid tapping performance compared to control vertex stimulation (C). $* P<$ 0.05 vs. WS or mean baseline, $+P<0.05$ vs. $\mathrm{PA}_{\mathrm{L}}$ and $\mathrm{V}_{\mathrm{s}}$.

Targeted stimulation of behaviourally-relevant interneuron populations may produce clearer behavioural effects.

\section{REFERENCES}

[1] R. Agostino, E. Iezzi, A. Suppa, A. Conte, A. Berardelli "Effects of intermittent theta-burst stimulation on practice-related changes in fast finger movements in healthy subjects", Eur. J. Neurosci., 28, 822-828, 2008.

[2] K. D'Ostilio et al. "Effect of coil orientation on strength-duration time constant and I-wave activation with controllable pulse parameter transcranial magnetic stimulation", Clin. Neurophysiol., 127, 675-683, 2016.

[3] V. Di Lazzaro \& J.C. Rothwell "Corticospinal activity evoked and modulated by non-invasive stimulation of the intact human motor cortex", J. Physiol., 592, 4115-4128, 2014

[4] J. Duque \& R.B. Ivry "Role of corticospinal suppression during motor preparation”, J. Neurosci., 19, 2013-2024, 2009.

[5] M. Hamada et al. "Two distinct interneuron circuits in human motor cortex are linked to different subsets of physiological and behavioral plasticity" J. Neurosci, 34, 12837-12849, 2014.

[6] Y-Z. Huang, M.J. Edwards, E. Rounis, K.P. Bhatia \& J. C. Rothwell, "Theta burst stimulation of the human motor cortex", Neuron, 45, 201-206, 2005.

[7] A.V. Peterchev, K. D’Ostilio, J.C. Rothwell \& D.L. Murphy, "Controllable pulse parameter transcranial magnetic stimulator with enhanced circuit topology and pulse shaping" J. Neural Eng., 11, $056023,2014$. 